\title{
An Intelligent Transportation Systems Architecture using Wireless Sensor Networks
}

\author{
Vivek Katiyar \\ Department of Computer \\ Science and Engineering \\ National Institute of Technology \\ Hamirpur, HP, INDIA
}

\author{
Prashant Kumar \\ Department of Computer \\ Science and Engineering \\ National Institute of Technology \\ Hamirpur, HP, INDIA
}

\author{
Narottam Chand \\ Department of Computer \\ Science and Engineering \\ National Institute of Technology \\ Hamirpur, HP, INDIA
}

\begin{abstract}
As the city road networks is growing day-by-day, the question of how to obtain information about the roads is becoming more and more challenging. In such an era Intelligent Transportation Systems (ITSs) has emerged as a key candidate that is benefited from the unique features and capabilities of Wireless Sensor Networks (WSNs) and Bluetooth technology. WSNs are composed of small tiny devices that work in autonomous manner to sense the surroundings. The Bluetooth protocol can be used for intervehicle communication among vehicles equipped with Bluetooth devices. ITSs are a state of art combination of transportation infrastructure and computer and information technology. ITSs can also resolve severe situations like traffic congestion and cope with emergency conditions like major accidents. This paper presents an efficient architecture that will increase the safety of road travel using the concepts of WSN and the Bluetooth protocol. We also discuss the ad-hoc network formation between vehicles and data exchange sensed by sensors. The simulation results show that Bluetooth and sensor networks can be used collaboratively to increase the safety of road travel.
\end{abstract}

\section{General Terms}

Algorithms

\section{Keywords}

Architecture, Bluetooth, Communication, Intelligent transport system, Wireless sensor networks

\section{INTRODUCTION}

With the advances in the technology of microelectromechanical system (MEMS), developments in wireless communications and wireless sensor networks (WSNs) have also emerged. WSNs are usually composed of small, low-cost devices that communicate wirelessly and have the capabilities of processing, sensing and storing. The nodes communicate wirelessly and often self-organize after being deployed in an ad hoc fashion. It is an infrastructure comprised of sensing (measuring), computing, and communication elements that gives the user the ability to instrument, observe, and react to events and phenomena in specified environment. Currently, WSNs are beginning to be deployed at an accelerated pace. It is not unreasonable to expect that in 5-10 years that the world will be covered with wireless sensor networks with access to them via the Internet. This can be considered as the Internet becoming a physical network. The development of WSNs was motivated by military applications such as battlefield surveillance and target tracking. Some typical applications of WSNs are tracking, monitoring and controlling. In a typical application a WSN is scattered in a region where it is meant to collect data through its sensor nodes. WSN are being used in many industrial and civilian application areas including industrial process monitoring and control [1, 2], machine health monitoring [3], environment and habitat monitoring, healthcare applications, home automation and traffic control[ $[1,4]$.

One of the major challenges that vehicle control and traffic management applications are facing is to know the position and speed of the vehicles on the road network in real-time is [5]. WSN received significant attention in the last decade and successful research put them in the forefront to answer this challenge [5]. WSN technology can help in the infrastructure development of ITS.

Vehicles equipped with Bluetooth radios can communicate wirelessly with each other. The California PATH program [10] has created a complete Autonomous Highway System (AHS) in which vehicles travel together in platoons. Although it is a demonstration of intelligent vehicle technologies, this requires major changes in the existing highway infrastructure to implement such a system. Forming platoons seems to be infeasible in real world because everyday traffic follows random patterns and this also depends on the type of vehicle and its driver.

Various governmentally-funded ITS projects have been launched in many countries like Canada [6], USA [7], Europe [8], Japan [9], Australia [10] and others. Furthermore, various projects have been funded by educational institutions, regional organizations and the industry to research on ITS. Examples include CAPTIV [11], SAFESPOT [12][13], PATH [14], FLEETNET [15][16][17], CVIS [18], TRACKSS [19] and MORYNE [20]. The growing interest in ITSs drove the development of many frameworks (like ART-Wise [21]) and standards (like WAVE (IEEE 802.11p) [22, 23] and CALM $[24,25])$. ITSs depended on traditional monitoring sensors including inductive loops, video cameras, ultrasonic sensors and radar [24].

\section{Key Technologies:}

The basic functionalities for WSN depends on the application, which in this case is ITS. In ITS, the most important features are the vehicle states, which includes position, speed and direction. Another possibility of feature may be classification of object whether it is a vehicle in traffic a car, a truck, or a bus. Some possible sensors for ITS include magneto resistive, light, pressure and videos that are visible or infrared.

As for the transmission part is concern, there are several wireless standards available. WSN can use some wireless technologies such as Bluetooth, ZigBee, Wireless LAN, WiMax and 3G. Range of communication, data throughput and power consumption are some of the properties that have 
to be put into considerations. $3 \mathrm{G}$ covers the longest range followed by WiMax, Wireless LAN, Bluetooth and Zigbee. Theoretically all those technologies are useful for ITS however it is all depend on the topology and implementation of the system.

ITSs generally require a high data rate for communication. ZigBee are generally used in spite of having a low data rate because they are very energy efficient. ZigBee is not very suitable for responsive, real-time communication systems because their data rate is as low as $20 \mathrm{kbps}$. ZigBee is so much energy efficient that it can run several years on a single set of batteries [26]. Although Bluetooth offers approximately 1 to $3 \mathrm{Mbps}$ and Wireless LAN have a maximum data transmission rate of $54 \mathrm{Mbps}$, but none of them as energyefficient as ZigBee.

Each sensor node needs an operating system to fill the gap between application and hardware, provides hardware abstraction and also capable of controlling hardware. TinyOS [26] is one of the de facto standards of operating system designed specifically for WSN. Its component library includes network protocols, distributed services, sensor drivers and data acquisition tools.

In this paper, we are proposing an efficient and reliable architecture for intelligent transport system with the help of WSN and ad-hoc networks formed among vehicle using Bluetooth. Bluetooth provides reliable communication between vehicles equipped with Bluetooth devices. Sensor nodes in WSN are deployed at important places to collect and provide information from/to vehicles. Vehicles communicate by forming an ad-hoc network and serve the base station so that coming vehicles can use this information. This is a more reliable ITS architecture compared to the previously proposed architectures.

The rest of the paper is organized as follows. In Section 2 we describe the design requirements for ITSs. Section 3 will describe the Bluetooth inter-vehicles communication model. This section shows how the ad-hoc network (aka piconet) is formed among vehicles to communicate and gather information. We discuss our proposed architecture in Section 4. Section 5 shows the analysis and simulation results. Lastly we conclude our paper in Section 6.

\section{DESIGH REQUIREMENTS}

Unique features of WSNs can help to benefit the diverse applications for efficient ITSs. Although design requirements for WSN architectures hold for any application, we may relax some of those requirements when dealing with Intelligent Transportation Systems:

\subsection{Network Topology}

Monitoring vehicles is one of the primary targets of an ITS. By having accurate information about the locations of each node, we can attain accurate information about the events taking place on the roads. Vehicles can also form an ad-hoc network with the help of Bluetooth to share the information collected by each other. Sensors are deployed on roadsides, or at known locations, eliminates the burden of implementing node localization algorithms. Due to stable environment and the availability of power resources, there is a good opportunity of minimizing the possibility of partitioning the topology of the network by minimizing node failures. So the change in topology will not be major concern and the design of routing protocols will be simple.

\subsection{Power consumption}

Sensor nodes are generally installed on vehicles or on roadsides for ITSs. Bluetooth devices and sensors installed in vehicles can use vehicles battery power to operate while the roadside sensors can be charged with the solar power. So we can think of no power constraints for WSNs in an ITS, resulting to handle more complex processing and complex computations. This leads to use of routing protocols having expensive computational requirements.

Power is the primary conservation in implementation of MAC and routing protocols. Other QoS factors are usually concerned as secondary. The applications of updating drivers of severe emergency, security, weather, or life threatening conditions are time-sensitive and can strongly benefit from QoS support in ITSs. Since sensors are limited in memory and processing capabilities, there is an upper limit on the performance to be achieved.

\subsection{Scalability and Network Costs}

Although WSNs are capable of handling a large number of sensor nodes. An efficient ITS is expected to cover hundreds of roads \& regions and millions of vehicles. This leads to the concern that how large the WSN will be to cover such a huge area. With traffic estimation, only a small fraction of the road systems will be measured and monitored by a WSN [27]. Traffic estimation can be use to estimate traffic data at locations where sensors are not deployed. This leads to significant drop in the deployment cost of the WSNs and traffic estimation can also support to make WSNs more scalable.

\subsection{Fault tolerance}

ITSs can be deployed in cities as well as rural areas. The ITSs are generally considered to be safe in urban areas from instable environmental conditions and thus sensor nodes failures will be minimized. This has its direct impact on prolonging the lifetime of the WSN, designing the different protocols and maintaining its topology.

\subsection{Security}

WSNs for ITS, such networks are soft target for intruders and attackers. In order to reflect a corrupt or fake image about the traffic or conditions on the roads one can manipulate data exchanged among sensor nodes and can cause a serious problem. As power is not such a bottleneck for such WSNs, we can implement sophisticated encryption algorithms to achieve higher levels of security.

\section{INTERVEHICLE COMMUNICATION MODEL USING BLUETOOTH}

A number of wireless communication standards have been proposed such as IEEE 802.11, Bluetooth, ZigBee etc. that can be used for communication among vehicles in ITSs. In this paper, we discuss the ITS that uses Bluetooth technology to form ad-hoc networks among vehicles in the traffic. Bluetooth is an open wireless technology standard for exchanging data over short distances from fixed and mobile devices and provide a high levels of security. Bluetooth has a raw bandwidth of $1 \mathrm{Mbps}$.

A Bluetooth device can discover other Bluetooth devices in its communication range by the process of inquiry. During the inquiry process same hop sequence is used for sending and listening. A sender can inquire more than one listener at a time. A sender unicast messages to a specific listener. Listener receives the message and send acknowledgement. In this way, listener becomes the slave and sender becomes the master. This process is called paging. Following this way, to 
communicate among themselves Bluetooth devices form piconets. There can be upto seven active slaves and one master in a piconet. Figure 1 shows vehicles in a piconet. If the number of slaves for a master exceeds to that limit, some slaves have to be parked. When master wants to communicate with a parked slave it have to first unpark it by parking some other active slave.

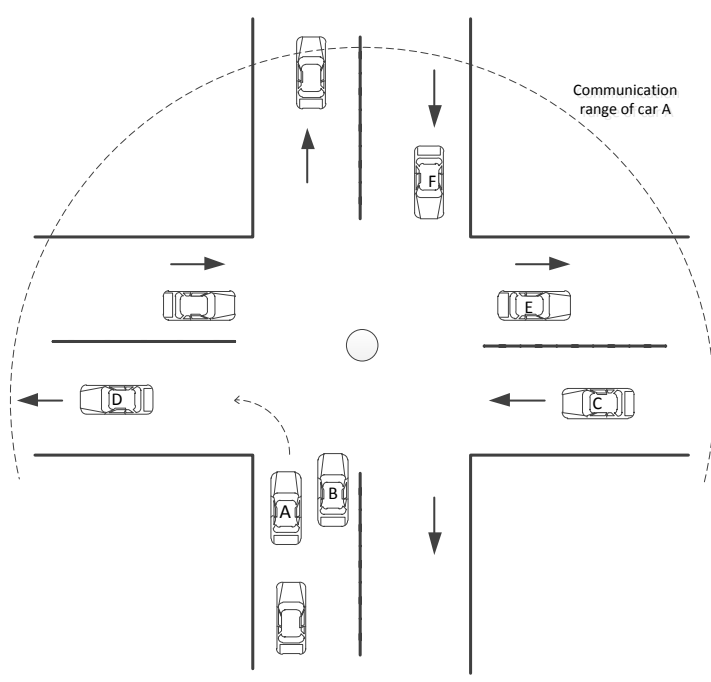

Figure 1. A traffic scenario at crossing.

A scatternet can be formed by joining two or more piconets. The Bluetooth standard allows a device to have multiple roles. It can be master in one piconet while it can be a slave in one or more other piconets at the same time. But, a device can be active only in one piconet. Slave devices can communicate only with the help of master. In ITS, by using such piconets \& scatternets and WSNs vehicles can communicate their sensed data with other vehicles in the network and can travel safely.

The following sub-section shows a typical traffic scenario where there may cause a potential accident. We will show how the potential accident can be avoided by sensors equipped in vehicles and WSNs that forms ITS.
A networked approach for communication among vehicles and WSNs can increase the level of safety. A traffic scenario of a road crossing in India is shown in Figure 1. If we assume that the signal for the horizontal lanes turned green, while the signal for the vertical lanes is red. Car A wants to make a left turn and car $\mathrm{C}$ wants to go straight from right to left. Neither car C nor car A sees each other due to the car B. Sensing range of car A is shown in the figure 1. It can sense all vehicles presented on that crossing and get the information. Car A only needs the information about car C, so to collect data in such scenario is critical task.

Wireless Sensor Networks deployed on that area will help for this reason. Sensor nodes deployed nearby region provide the information about car $\mathrm{C}$ to car A before reaching to the crossing. In this way either car A triggers a message to car $\mathrm{C}$ to cross or let it cross first. Car A does not need to communicate to car D because it already crossed and its information is irrelevant.

Our proposed architecture provide a mechanism to communicate with vehicles in real time with Bluetooth, depending upon data collected from sensor network deployed in that region. In our proposed architecture we deploy the powerful sensor nodes also called as Base Station (BS) at every important area like crossing, bridges, one-ways etc. vehicles can get information about the traffic and road conditions from BS. They can also communicate with each other by forming an ah-hoc network if no such BS is available. Proposed approach is more reliable to the previous approaches.

\section{PROPOSED ARCHITECTURE}

Low power standards like ZigBee are not suitable for ITS because vehicles are running in speed. Sensor equipped in vehicles does not suffer from problem of power as they can be charged by vehicle battery. As described base stations are deployed at important places only, they get information from the vehicles running on the road. They will optimize information by efficient data aggregation and serve it to the vehicle in need.

In our proposed approach ITS is formed with the help of WSN and ad-hoc network formed by Bluetooth devices attached to vehicles.

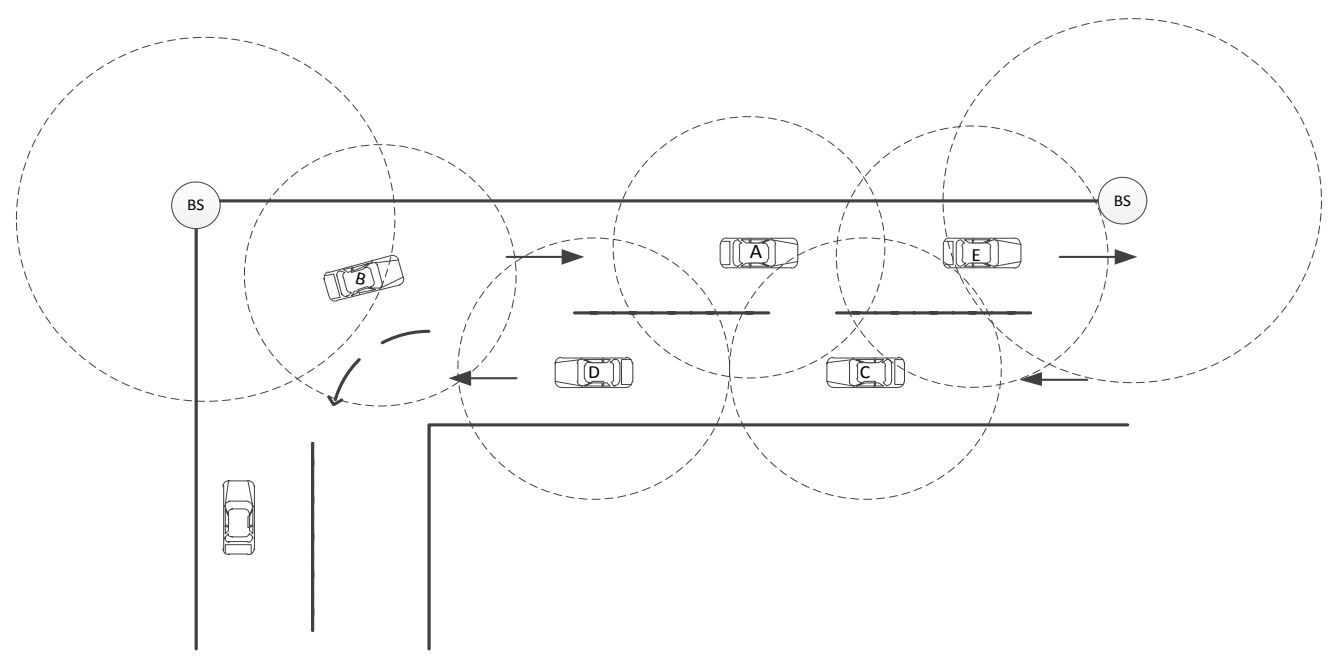

Figure 2. Communication model between vehicles and WSN-BS.

If we consider the scenario shown in Figure 2, base stations will have the information about road conditions that will previously provided by passing vehicles. In this way, cars A, $\mathrm{B}$ and $\mathrm{E}$ can get information from the one BS and store some 
information to the other BS at the same time. BS are supposed to monitor up to 200 vehicles and sense $100 \mathrm{~m}$. BS are powered with solar power.

In the following subsection we describe proposed algorithm. Vehicles running on the road first search for the base station, if it find the BS it communicate with it otherwise it seeks the vehicles equipped with Bluetooth devices and communicate. In this way, there are two types of communications to be considered between WSN-BS and vehicles running on the roads as shown in Figure 2.

\subsection{Communication between vehicles moving on the road}

If the vehicle does not find the BS it communicates with vehicles in its communication range by forming piconet in following manner. If we consider two cars want to communicate with each other. As describe in the previous section one of the cars searches for vehicles in its range. Any vehicle can communicate up to seven vehicles at a time. Car $\mathrm{A}$ can find cars D, C and $\mathrm{E}$ in its communication range. They form a piconet and car A can send message to any of them. Since car E can communicate with BS, car A will communicate to BS with help of car E.

\section{Communication between car A and car $E$}

\section{Car A to Car E:}

1. Car E is detected by car A within its transmission range.

2. A connection request is sent by car A to car E.

\section{Car E to Car A:}

3. An ACK is sent by car E to car A. It also includes its ID.

Car A to Car E:

4. Information regarding the state of the roads and traffic density of all the road segments that are in the destination path of car A is provided by car E.

Car A to Car E:

5. If car A reaches out of the coverage limit of car E and vice versa, the connection ends.

\subsection{Communications between static sensors in a WSN and vehicles moving on the road}

\section{Car E to WSN-BS:}

1. As the Car E detects a WSN-BS within its transmission range.

2. Car E sends a connection request to the WSN-BS. Car E also sends its ID.

\section{WSN-BS to Car E:}

3. The WSN-BS sends back an ACK to the Car E.

\section{Car E to WSN-BS:}

4. Car E request to WSN-BS about the road condition regarding all segments that are in the path along its destination. Car E also sends to the WSN-BS road information which was previously gathered from other vehicles or other WSN. The WSN updates that information (if newer) regarding the state of the roads for vehicles coming after car E.

\section{WSN-BS to Car E:}

5. If car E reaches out of the coverage limit of WSN-BS, the connection ends.

In this way, vehicles update the information at WSN-BS that is utilized by the vehicles coming afterwards. So this can be thought of as an intelligent network of the vehicle, for the vehicles, by the vehicles.

\section{SIMULATION RESULTS}

In this section we show that how performance is evaluated for proposed architecture. here we has assumed that wireless sensor nodes are attached at every important location on an average distance of $100 \mathrm{~m}$. vehicles running on road forms an ad-hoc networks between vehicles. Vehicles cooperatively update the data on the WSN-BS so that it can provide information to the vehicles in need. Vehicles are communicating using Bluetooth. One thing to be noticed here is that reliability is bigger issue than energy efficiency for such architectures because WSN-BSs and vehicles have sufficient energy resources.

The proposed architecture is implemented and simulated using Omnet++ 4.1. To evaluate the performance we consider number of packets successfully delivered and delay as performance metrics.

We consider the scenario same as in Figure 2. There are two WSN-BS situated at a distance of $100 \mathrm{~m}$ and five cars are running between them. Cars have average communication range of 15 meter. Data source rate is $1 \mathrm{Mbps}$ and MAC protocol we are considering is IEEE802.11b. We are evaluating proposed architecture under DSR (Distance Source Routing) [28], a well known routing protocol for ad-hoc networks.

\subsection{Successful delivery of data packets}

Here we assume that vehicles are equipped with sensors with variable range between $10-20 \mathrm{~m}$. The average range can be considered as $15 \mathrm{~m}$. Successful delivery of data packets will be affected by range. The variation is clear from Figure 3 . We perform simulation for two values of packet sizes (500 bytes and 1000 bytes). As it is clear from Figure 3 the percentage of successfully delivered packets increases for large packet size.

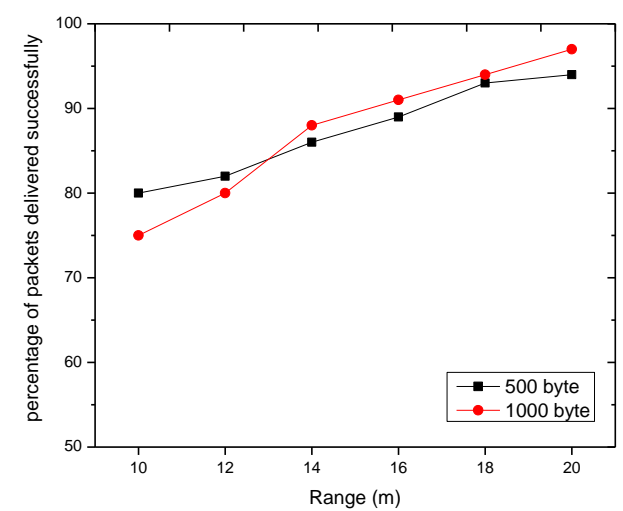

Figure 3. Impact of sensing range on successful delivery of data packets.

\subsection{Delay in delivery of data packets}

Vehicles may have speeds between $40-100 \mathrm{~km} / \mathrm{h}$. The average speed we considering as $60 \mathrm{~km} / \mathrm{h}$. It is clear from Figure 4 that least delay in delivery of data packet can be seen at this speed. When the vehicle is too slow then delay will be high and same if it is too fast delay will also be high. At any speed delay for big packet will be high. Here we are taking packets of size 500 bytes and 1000 bytes. 


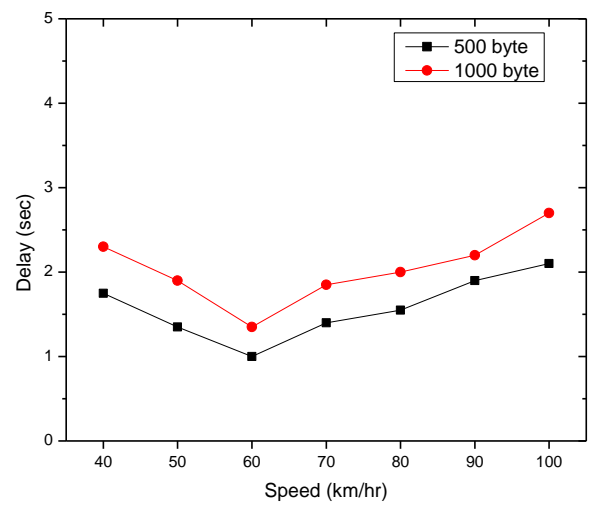

Figure 4. Impact of vehicle speed on delay in delivery of data packets.

\section{CONCLUSION}

In this paper we addressed the approach of sensor networks to implement an efficient ITS that increase the safety of road travel. To deploy an reliable ITS, we proposed to deploy the WSN-BSs at important places to collect and provide information to the vehicles. We showed that the exchange of sensed data among vehicles can be beneficially used to avoid accidents. In this paper, we discussed some important design parameters that have a huge effect on the design of ITSs architecture. We have also reviewed some key technologies for communication. We drive an algorithm that provide an reliable ITS for the society.

\section{REFERENCES}

[1]. R. Kay and F. Mattern, "The Design Space of Wireless Sensor Networks", IEEE Wireless Communications Vol. 11, No. 6, December 2004, 54-61.

[2]. T. Haenselmann, Sensornetworks. GFDL Wireless Sensor Network textbook. http://pi4.informatik.unimannheim.de/ haensel/sn_book. Retrieved 2006-08-29.

[3]. A. Tiwari, P. Ballal and F.L. Lewis, "Energy-efficient wireless sensor network design and implementation for condition-based maintenance", ACM Transactions on Sensor Networks (TOSN), Vol. 3, No. 1, 2007, 1-23.

[4]. S. Hadim and N. Mohamed, "Middleware: middleware challenges and approaches for wireless sensor networks," Distributed Systems Online, IEEE, Vol.7, No.3, March 2006, 1-23.

[5]. J. Sijs, Z. Papp and P.P.J. Bosch, "Vehicle Motion-StateEstimation Using Distributed Sensing," IEEE Intelligent Vehicles Symposium Eindhoven University of Technology Eindhoven, June 4-6, 2008, 458-463.

[6]. Intelligent Transportation Systems Society of Canada: http://www.itscanada.ca/english/index.htm, last accessed on Dec. 20th, 2010.

[7]. Research and Innovative Technology Administration (RITA), Intelligent Transportation Systems: www.its.dot.gov, last accessed on Dec. 20th, 2010.

[8]. ERTICO - ITS Europe: www.ertico.com, last accessed on Dec. 20th, 2010.
[9]. Ministry of Land, Infrastructure, Transport and Tourism in Japan: www.mlit.go.jp/index_e.html, last accessed on Dec. 20th, 2010.

[10].Intelligent Transportation Systems in Australia: www.itsaustralia.com.au, last accessed on Dec. 20th, 2010.

[11].O. Berder et al., "Cooperative communications between vehicles and intelligent road signs", Proceedings of the 8th International Conference on ITS Telecommunications (ITST'08), Vol. 1, Oct. 2008, 121-126,

[12].SAFESPOT Integrated Project: www.safespot-eu.org, last accessed on Dec. 20th, 2010.

[13].R. Brignolo, "The SAFESPOT Integrated Project - Cooperative Systems for Road Safety", TRA Conference Goteborg, Jun. 2006.

[14].PATH project: www.path.berkeley.edu, last accessed on Dec. 20th, 2010.

[15].FleetNet project: www.et2.tu-harburg.de/fleetnet/, last accessed on Aug. 20th, 2009

[16].W. Franz, R. Eberhardt, and T. Luckenbach, "FleetNet Internet on the Road", the Proceedings of the 8th World Congress on Intelligent Transportation Systems, Sidney, Australia, 2001.

[17].H. Hartenstein, B. Bochow, A. Ebner, M. Lott, M Radimirsch, D. Vollmer, "Position-Aware Ad Hoc Wireless Networks for Inter-Vehicle Communications: The FleetNet Project", the Proceedings of the ACM Symposium on Mobile Ad Hoc Networking \& Computing (MobiHoc'01), Long Beach, California, 2001, 233-245

[18].CVIS project: www.cvisproject.org, last accessed on Dec. 20th, 2010.

[19].TRACKSS project: www.trackss.net, last accessed on Dec. 20th, 2010.

[20].MORYNE project: www.fp6-moryne.org, last accessed on Dec. 20th, 2010

[21].ART-Wise framework: www.hurray.isep.ipp.pt/art-wise, last accessed on Dec. 20th, 2010.

[22].R. Uzcategui, G. Acosta-Marum, "WAVE: A Tutorial", IEEE Communications Magazine, Vol. 47, No. 5, , May $2009,126-133$

[23].IEEE 1609 Family Standard of Wireless Access in Vehicular Environments (WAVE)

[24].CALM: www.calm.hu, last accessed on Dec. 20th, 2010.

[25].ISO/DIS 21210 Intelligent Transport Systems Communications Access for Land Mobile (CALM) Networking Protocols.

[26].Kazem Sohraby, Daniel Minoli. Wireless Sensor Network. 2007

[27].M. Tubaishat, P. Zhuang, Q. Qi, and Y. Shang, "Wireless Sensor Networks in Intelligent transportation Systems," Wireless Communications \& Mobile Computing, Vol. 9, No. 3, 2009, 287-302.

[28].The Dynamic Source Routing Protocol (DSR). DOI=http://www.rfc-editor.org/rfc/rfc4728.txt 